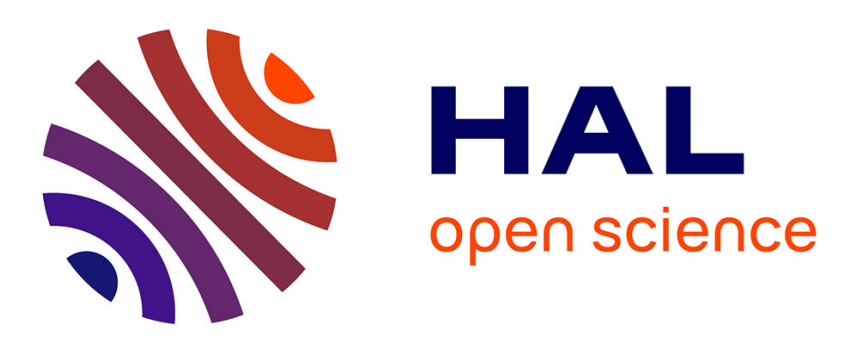

\title{
A Bayesian Model for Mobility Prediction in Wireless Sensor Networks
}

Fatma Somaa, Cédric Adjih, Inès El Korbi, Leila Azouz Saidane

\section{To cite this version:}

Fatma Somaa, Cédric Adjih, Inès El Korbi, Leila Azouz Saidane. A Bayesian Model for Mobility Prediction in Wireless Sensor Networks. 5th IFIP International Conference on Performance Evaluation and Modeling in Wired and Wireless Networks (PEMWN 2016), Nov 2016, Paris, France. hal01405277

\section{HAL Id: hal-01405277 \\ https://hal.inria.fr/hal-01405277}

Submitted on 29 Nov 2016

HAL is a multi-disciplinary open access archive for the deposit and dissemination of scientific research documents, whether they are published or not. The documents may come from teaching and research institutions in France or abroad, or from public or private research centers.
L'archive ouverte pluridisciplinaire HAL, est destinée au dépôt et à la diffusion de documents scientifiques de niveau recherche, publiés ou non, émanant des établissements d'enseignement et de recherche français ou étrangers, des laboratoires publics ou privés. 


\title{
A Bayesian Model for Mobility Prediction in Wireless Sensor Networks
}

\author{
Fatma Somaa*, Cédric Adjih ${ }^{\dagger}$, Inès El Korbi* and Leila Azouz Saidane* \\ * National School of Computer Science, University of Manouba, 2010 Tunisia \\ ${ }^{\dagger}$ Inria Saclay-Île-de-France, Palaiseau, France \\ * \{ines.korbi, leila.saidane $\} @$ ensi.rnu.tn, fatma.somaa@sesame.com.tn \\ †cedric.adjih@inria.fr
}

\begin{abstract}
Wireless sensor networks (WSN) have specific features such as low transmission range, stringent energy consumption constraints, limited memory and processing power. For this reason, tailored protocols have been proposed, optimizing network protocols with respect to various assumptions: one commonly exploited property of WSN is the stability of the topology due to permanent installation of sensor nodes. However, in some applications, some of the wireless sensor nodes might be mobile, for instance when they are associated with users. In that case, specific extensions of WSN protocols need to be designed. Then a first step is the characterization of nodes' mobility. This is the focus of this article: we propose a general method of mobility estimation for wireless sensor networks. Namely, using a Bayesian framework, we derive a mobility prediction model to estimate the node velocity (starting from no knowledge) from observed events. In this article, we focus on events represented by the most minimal information: mere observation of link duration with neighbors. Simulations illustrate that, even with such limited information, the mobility can be well inferred, and results show the good performance of the method.
\end{abstract}

\section{INTRODUCTION}

Wireless sensor networks are integrated in very diverse applications to achieve several purposes such as monitoring, detecting anomalous events and various measurement collection tasks. A key feature of such networks is their facility of deployment and their auto-adaptivity. However, due to the limited capacities of wireless sensor nodes, auto-adaptivity sometimes comes at the price of lesser generality: many WSNs are not integrating routing protocols relying on more complete information such as in mobile ad-hoc networks (MANETs), or wireless mesh networks.

For instance, if we consider RPL (IPv6 Routing Protocol for Low power and Lossy Networks) [21], the IETF routing protocol for WSNs, RPL is designed in such a way to adapt to WSN constraints (low energy resource, low memory processing capabilities, etc.). But RPL is still inefficient faced to nodes' mobility and its performance degrades consequently [24].

Some works [22], [23] have been proposed for monitoring and detecting neighboring stable links but at the expense of extra resource consumption. Some other schemes [24], [25] have been proposed to enhance the RPL behavior. But their contribution remains limited since they require several changes to native RPL. The resulting schemes, even improving the WSN behavior in case of nodes' mobility, largely deviate the native RPL behavior.

In general, WSN nodes are assumed to be static, and performance may degrade if mobile nodes are present without being taken into account. Therefore, first, characterizing node mobility, and second, integrating the mobility into WSN routing protocols is necessary in this scenario. The topic of mobility is explored in this article: we propose a method to automatically detect and estimate the nodes' mobility in WSNs. The key idea of this paper is to accurately predict the sensor nodes' movement (velocity) without pre-knowledge of any mobility information within the sensor node (current position/speed).

A straightforward way to derive sensor nodes' mobility is to equip each sensor node with a specific equipment (e.g. GPS Global Positioning System) to estimate the node's mobility [2], [3], [11]. As we could expect, using such techniques are not universally applicable (GPS not embedded for cost reasons, limited GPS coverage, limited GPS precision, ... ).

Other techniques, based on sensor nodes' mobility estimation propose new metrics or new extensions for routing protocols [1], [4], [10], [6], sometimes relying on analytical models [16], [14], [5], [19].

The key difference between our approach and the above mentioned techniques is that, we are deliberately making minimal assumptions and intend to propose the most general solution possible. For instance, sensor nodes have no knowledge of their initial positions/velocities. Nodes discover dynamically existing and broken links. The proposed framework will allow, given an initial random distribution of the sensor nodes' speed, to rapidly converge to the real distribution using Bayesian inference [29]. The strength of Bayesian inference is that a unique event (a broken link between two nodes namely $U_{i}$ and $U_{j}$ ) allows refining the velocity/mobility prediction of all $U_{i}$ and $U_{j}$ neighbors. Therefore, the Bayesian inference allows collaborating nodes to quickly converge to a correct mobility prediction despite having initial arbitrary information about their speed values.

In this paper, we present the different steps of the proposed Bayesian model to predict the sensor node's mobility. Then we evaluate the convergence of estimated speed values to the real values, through simulations.

The rest of the paper is organized as follows. In Section II, we discuss the literature related to mobility prediction in WSNs. Section III defines our mobility prediction model based 
on Bayesian inference. In Section IV, we simplify our model (to make it even more suitable for the WSN context) and evaluate its performance. Finally, Section V concludes the paper and present directions for future work.

\section{BACKGROUND}

In this paper we intend to propose a novel mobility prediction scheme for WSNs based on Bayesian inference [29]. As mentioned above, the sensor nodes' mobility in WSNs is a critical issue and if no actions are taken with respect to the sensor nodes' movement, the whole network performance will be considerably altered when the sensors effectively move.

Hence, we propose in this section to review the works in literature related to sensor nodes' mobility and mobility prediction in WSNs. According to the technique used to predict the sensors' movement, the related studies can be classified in three categories: mobility estimation techniques, routing based mobility prediction techniques and mobility prediction analytical models.

\section{A. Mobility estimation techniques}

One simple way to estimate the sensor nodes' mobility is to equip them with GPS receivers as proposed in [2], [3]. GPS gives the absolute coordinates of a mobile sensor at the expense of cost and energy.

Moreover GPS may suffer from frequent satellite disconnections in indoor environments. To estimate nodes' mobility, methods uses the Received Signal Strength (RSS) to measure the power of a received signal [11] as an indicator of the distance between two nodes. However, these techniques might be computationally expensive (time, CPU and memory). More importantly, they require perfect modelling of the wireless channel: the obtained values will fluctuate according to multipath effects, fading, or limited temporal coherence (due to the presence of obstacles for example or in mobile environment) leading to imprecise values. A common issue in such mobility estimation techniques using sensors (RSSI, ranging devices, ...) is that they often rely on some hypothesis (such as perfect models, or at least good characterization of the stochastic component of the measured values, e.g. noise, error, variance). In real implementations, it might happen that some hypothesis are not verified.

\section{B. Routing based mobility prediction techniques}

In this paragraph, we discuss the different works using the routing metric to predict the sensor nodes' mobility in wireless networks. A first example is [1], using a link stability metric as a mobility indicator between two nodes. This metric is defined as the number of successive beacons. The mobility prediction is based on the past received values. However, the lost beacon frames used to predict the link stability provide false predicted values due to obstacles, collisions, etc.

[10] proposes a modified AODV (Ad hoc On demand Distant Vector) routing scheme to predict the neighbors' mobility in MANET (Mobile Ad hoc NETwork by calculating the effective communication distance (the distance between two nodes that allows them to directly communicate). In fact, the update of the effective communication distance is triggered when the real distance between nodes is higher than the estimated distance. This approach has some disadvantages such as requiring a centralized agent with a permanent power or periodic calculation of the received power.

\section{Mobility prediction analytical models}

In this paragraph, we discuss the analytical works modelling the sensor nodes' mobility in WSNs. Many approaches are based on Markov chain paradigm. In [16], links lifetime is considered as the sum of two states' Markov model: the link availability when the velocities of the two nodes keep unchanged during a period $T_{p}$ and the second state represents the case where velocity changes. Accordingly, the probability that the link is broken is divided into two states. This approach requires the knowledge of the nodes' velocity. The mobility prediction model in [19] is based on the Markov assumption: the future depends only on the present state. Hence, the state must contain sufficient information to get a precise system behavior prediction.

The models presented in [14] are an inspiration for our work: they characterize the probabilistic properties of observed events (link duration, number of neighbors, ...), with respect to node speeds and directions (considered as random variables) under some assumption. However they do not go the extra step of using this as input for estimating the random variables (mobility parameters).

Recently, [5] focus on Bayesian model to predict mobility in WSNs. It uses metadata to predict the network behavior. This approach is very difficult to implement because requires self configured nodes and needs a coordinator to collect the results. In [5], the authors proposed a Bayesian model to predict mobility in ad hoc networks hence helping routing protocols to avoid broadcasting request messages in mobile regions. Therefore, the model classifies the nodes' speed in three categories (low, medium and high) based on two estimated quantities Average Encounter Rate (AER), given the average number of new encounters experienced in a duration $\mathrm{T}$ and the node's degree known as the number of one node's neighbors. Two nodes encounter each other when the distance between them becomes smaller than the communication range. Even using Bayesian inference, [5] only gives a rough prediction of nodes' mobility without deriving any accurate information about effective speed values.

\section{BAYESIAN MODEL FOR NODE SPEED AND LINK DURATION ESTIMATION}

In this section, we present our Bayesian inference framework for the mobility prediction in WSNs. WSNs may contain a certain number of mobile sensor nodes depending on the network deployment context. An accurate prediction of sensor nodes' mobility can obviously aid the network to reactively adapt to such topological changes.

We propose a framework to predict sensor nodes' speed using a Bayesian inference framework [29]. In short: a node 
maintains an estimate of its speed as a distribution (e.g. probability that the speed equals such and such value). This distribution represents the best knownledge/estimate that a node possesses of its own speed (at a given moment). Bayesian inference consists in having sensor nodes to updating their speed estimates with each encountered event. Starting from any given unknown initial velocity distribution, the estimate will be gradually updated towards the actual values, assuming that the speed stays constant.

With Bayesian inference, convergence is ensured hence avoiding non-trivial computational issues we may encounter with some other models [26]. Moreover, two additional properties are: implicit robustness, because the inference is constantly refining estimates, thus any noise or errors would fade gradually with further estimates; and extensibility, with the possibility of taking into consideration additional events (for instance, an event "observed variation in accelerometer sensor").

\section{A. Overview and Assumptions}

We deliberately consider that sensor nodes have no information about their positions, velocities, speed variation, etc. The only information that allows predicting accurate speed values is the occurrence of the particular events. In this article, we consider the case of events "broken links" caused by sensor nodes' movement (nodes get out of range). We detail a new framework based on Bayesian inference to derive real sensor nodes' speeds upon the occurrence of subsequent events (here, "broken links" events). Hereafter, we present the key assumptions we adopt in the rest of our study:

- Assumption 1: The sensor network is composed of both mobile and fixed nodes.

- Assumption 2: Sensor nodes have limited resources and have no information to derive their speed values (GPS, accelerometer, ...). Notice that when such additional source of information are available, they could be easily integrated in the Bayesian inference as additional events.

- Assumption 3: Sensor nodes' velocity magnitudes are constant over the time. Sensor nodes can however change direction.

- Assumption 4: We assume that the sensor nodes' directions are independently distributed [20].

In the following, we introduce the main notations:

- Let $\mathcal{E}$ be the particular event representing link duration before break between nodes $U_{1}$ and $U_{2} ; \mathcal{E}=\{$ a link between $U_{1}$ and $U_{2}$ lasted for $\left.\tau\right\}$. We denote $p_{\tau}(t)$ the associated probability density function (pdf) of link duration between two given nodes in the network.

- We also define $p_{i}\left(v_{i}\right)$, the pdf of the node $U_{i}$ speed. $p_{i}\left(v_{i}\right)$ is called the prior speed distribution of the node $U_{i}$ (prior to the event $\mathcal{E}$ ).

- Similarily, we have $p_{i, j}\left(v_{i}, v_{j}\right)$ the prior joint speed distribution.

- We also use the notation $p_{\ldots}^{+}(\ldots)$ for all the posterior distributions (after the event $\mathcal{E}$ ).

\section{B. General Bayesian Inference}

Exploiting the network dynamics, Bayesian inference allows updating posterior sensor nodes speed distributions using Bayes' rule. We consider a two nodes' scenario $U_{1}$ and $U_{2}$ having respective speeds $v_{1}$ and $v_{2}$, which were neighbor, and just observed the event $\mathcal{E}=$ their link has broken. Then, using Bayes' rule, we have:

$$
\begin{aligned}
& P\left(U_{1} \text { has a speed } v_{1} \text { and } U_{2} \text { has a speed } v_{2} \mid \text { event } \mathcal{E}\right) \\
= & P\left(\text { event } \mathcal{E} \mid U_{1} \text { has a speed } v_{1} \text { and } U_{2} \text { has a speed } v_{2}\right) \\
& \times \frac{P\left(U_{1} \text { has a speed } v_{1} \text { and } U_{2} \text { has a speed } v_{2}\right)}{\operatorname{Pr}(\mathcal{E})}
\end{aligned}
$$

where $\operatorname{Pr}(\mathcal{E})$ is the event $\mathcal{E}$ probability. With the model notations given in paragraph III-A, the posterior speed joint distribution of $v_{1}$ and $v_{2}$, resulting from the prior joint distribution $p_{1,2}\left(v_{1}, v_{2}\right)$ and the observed link broken event $\mathcal{E}$, is:

$$
p_{1,2}^{+}\left(v_{1}, v_{2} \mid \mathcal{E}\right)=\frac{1}{\operatorname{Pr}(\mathcal{E})} p_{\tau}\left(\mathcal{E} \mid v_{1}, v_{2}\right) p_{1,2}\left(v_{1}, v_{2}\right)
$$

Moreover, using the assumption 4, related to the independence of speeds $v_{1}$ and $v_{2}$, we have

$$
p_{1,2}\left(v_{1}, v_{2}\right)=p_{1}\left(v_{1}\right) \times p_{2}\left(v_{2}\right)
$$

As common in the Bayesian inference, $\operatorname{Pr}(\mathcal{E})$ is a normalizing constant: it can be ignored, under condition to later normalize posterior distributions (e.g. $\int p=1$ ). After this step, the independence property between $v_{1}$ and $v_{2}$ may not be still valid after inference, especially if nodes become later neighbors again, but as an approximation, we will ignore it, and use only the definition:

$$
p_{1}^{+}\left(v_{1} \mid \mathcal{E}\right)=\int_{v_{2}=0}^{+\infty} p_{1,2}^{+}\left(v_{1}, v_{2} \mid \mathcal{E}\right) d v_{2}
$$

From equations (2), (3) and (4) we obtain:

$$
p_{1}^{+}\left(v_{1} \mid \mathcal{E}\right) \propto p_{1}\left(v_{1}\right) \int_{v_{2}=0}^{\infty} p_{\tau}\left(t \mid v_{1}, v_{2}\right) p_{2}\left(v_{2}\right) d v_{2}
$$

\section{Conditional Link Duration Distribution}

In this article, the events considered are link breakage, and from inference in equation (5), deriving posterior speed distributions depend on the quantity $p_{\tau}\left(t \mid v_{1}, v_{2}\right)$ : the probability that a link lasts for a duration $t$ given prior speed distributions $v_{1}$ and $v_{2}$. Link duration is a random variable because the angle between the nodes is not known (supposed uniform in $[0,2 \pi])$. To evaluate the link duration, a good starting point is [13]. Other studies focused on link dynamics using different approaches (GPS [27], empirical residual link lifetime [28], etc.). Despite its accuracy, [13] introduces two relative angles $\alpha$ and $\beta$ to derive links' duration whereas only the "minimum distance" between the two nodes and the absolute value of the relative speed are needed. Hence, we propose here to derive a simple expression of link duration using the property: the "minimum distance during their encounter" between two 
nodes (that get in range of each other) is uniformly distributed in $[-R, R][13]$.

We first start with a normalized/unit parameters assuming that $v=1$ and the radio range $R=1$. We denote by $p_{\bar{\tau}}(t)$ the pdf of link duration with unit parameters. Let $U_{1}$ and $U_{2}$ be the nodes of the link ( $U_{1}$ has speed $0, U_{2}$ has speed $v$ ). To simplify the notation, without loss of generality, we assume that $U_{2}$ is moving horizontally. Let $y$ be the closest distance between $U_{1}$ and $U_{2}$ when they are in the communication range of each other. We assume that $y$ is an instance of a random variable $Y$ which is uniformly distributed in $[0,1]$ with density $p_{Y}^{*}(y)=1$. The relation between $Y$ and the duration $t$ is given by $t=f(y)=2 \sqrt{1-y^{2}}$. As in [18], we define:

$$
\left.p_{\bar{\tau}}(t)=\left|\frac{1}{\left.f^{\prime-1}(t)\right)}\right| p_{Y}\left(f^{-1}(t)\right)=\mid 1 / f^{\prime-1}(t)\right) \mid
$$

After calculating $f^{\prime}(x)$ and $f^{-1}(x)$, we get:

$$
p_{\bar{\tau}}(t)=\frac{t}{2 \sqrt{4-t^{2}}}
$$

Now, if we remove the assumption about $v$ and $R$, we have $\tau_{\text {actual }}=\frac{R}{v} \tau_{\text {normalized }}$. Thus we apply the change of variable $\tau=g(\bar{\tau})=\frac{R}{v} \bar{\tau}$

$$
p_{\tau}(t)=\frac{1}{\left.g^{\prime-1}(t)\right)} p_{\bar{\tau}}\left(g^{-1}(t)\right)=\frac{v}{R} p_{\bar{\tau}}\left(\frac{v}{R} t\right)
$$

This value is only defined when $0 \leq \frac{v}{R} t<2$, in other words $t<\frac{2 R}{v}$. In the following, we assume that $R=1$ and $v$ will be expressed in the following units: "radio-range per unit time" (instead of "unit distance per unit time"). Then:

$$
p_{\tau}(t)=v p_{\bar{\tau}}(v t)
$$

The speeds $v_{1}$ and $v_{2}$ result in a relative speed $v$ equal to $v=h(\theta)=\sqrt{v_{1}^{2}+v_{2}^{2}-2 v_{1} v_{2} \cos (\theta)}$, where $\theta$ is the angle between the nodes and is uniformly distributed in $[0,2 \pi]$. Using the same computations as in (6) and (8), we have for $v \in]\left|v_{1}-v_{2}\right|, v_{1}+v_{2}[:$

$$
\begin{aligned}
p_{V}\left(v \mid v_{1}, v_{2}\right) & =2\left|\frac{1}{\left.h^{\prime-1}(v)\right)} p_{\theta}\left(h^{-1}(v)\right)\right| \\
& =\frac{2 v}{\pi \sqrt{4 v_{1}^{2} v_{2}^{2}-\left(v_{1}^{2}+v_{2}^{2}-v^{2}\right)^{2}}}
\end{aligned}
$$

where $h^{\prime}(\theta)=\frac{v_{1} v_{2} \sin (\theta)}{\sqrt{v_{1}^{2}+v_{2}^{2}-2 v_{1} v_{2} \cos (\theta)}}$ and

$h^{-1}(v)=\arccos \left(\frac{v_{1}^{2}+v_{2}^{2}-v^{2}}{2 v_{1} v_{2}}\right)$ in the range $[0, \theta]$ and another one in the range $[\pi, 2 \pi]: 2 \pi-h^{-1}(v)$.

We can then express the conditional probability $p_{\tau}\left(t \mid v_{1}, v_{2}\right)$ that a link lasts for a duration $\tau$ when nodes have speeds $v_{1}$ and $v_{2}$. Notice that:

- The minimum possible relative speed is: $\left|v_{1}-v_{2}\right|$ (two nodes in the same direction).

- The maximum possible relative speed is: $v_{1}+v_{2}$ (two nodes crossing each other in the opposite direction).
- The maximum possible relative speed that could result in a link of duration $t$, is $v=\frac{2}{t}$.

We denote $v_{\ell}\left(v_{1}, v_{2}, t\right)=\min \left(v_{1}+v_{2}, \frac{2}{t}\right)$ the maximum relative speed given $v_{1}$ and $v_{2}$ that could possibly yield a link of duration $t$.

$$
\begin{aligned}
& p_{\tau}\left(t \mid v_{1}, v_{2}\right)=0 \text { if } \frac{2}{\left|v_{1}-v_{2}\right|}<t, \text { otherwise : } \\
& p_{\tau}\left(t \mid v_{1}, v_{2}\right)=\int_{v} p_{\tau}(t \mid v) p_{V}\left(v \mid v_{1}, v_{2}\right) d v \\
& =\int_{v=\left|v_{1}-v_{2}\right|}^{v=v_{\ell}\left(v_{1}, v_{2}, t\right)} \frac{v^{2} t}{2 \sqrt{4-v^{2} t^{2}}} \frac{2 v}{\pi \sqrt{4 v_{1}^{2} v_{2}^{2}-\left(v_{1}^{2}+v_{2}^{2}-v^{2}\right)^{2}}} d v \\
& =\int_{v=\left|v_{1}-v_{2}\right|}^{v=v_{\ell}\left(v_{1}, v_{2}, t\right)} \frac{v^{3} t}{\pi \sqrt{\left(4-v^{2} t^{2}\right)\left(4 v_{1}^{2} v_{2}^{2}-\left(v_{1}^{2}+v_{2}^{2}-v^{2}\right)^{2}\right)}} d v
\end{aligned}
$$

The expression (11) can be simplified through use of elliptic integrals [19]. This also allows faster computation for evaluating (11). We introduce the following quantities: $\alpha=\left(v_{1} v_{2}\right)^{2}$, $\beta^{*}=\left(v_{1}+v_{2}\right)^{2}, \gamma^{*}=\frac{4}{t^{2}}, \beta=\min \left(\beta^{*}, \gamma^{*}\right)$ and $\gamma=$ $\max \left(\beta^{*}, \gamma^{*}\right)$. Then, if $\beta \leq \alpha$, then $p\left(t \mid v_{1}, v_{2}\right)=0$, and otherwise:

$$
p_{\tau}\left(t \mid v_{1}, v_{2}\right)=\frac{\left(w^{2}+\alpha\right) K(k)-w^{2} E(k)}{\pi w}
$$

where $w=\sqrt{\gamma-\alpha}, k=\frac{\sqrt{\beta-\alpha}}{w}, E$ is the complete integral of the second kind and $K$ is the complete integral of the first kind in Jacobi form [19].

\section{The Bayesian Inference Implementation}

To implement the Bayesian inference model on the sensor network, we consider that sensor nodes periodically exchange Hello beacons to detect links' expiration. Then Bayesian inference can be performed with discretized quantities: each node $U_{i}$ maintains a vector $V_{i}$ of $n$ discrete velocity values representing its discretized pdf and initialized it with random distribution. Upon the occurrence of a link expiration event $\mathcal{E}$ between two nodes $U_{1}$ and $U_{2}$, the node $U_{1}$ evaluates the matrix $M\left(\tau_{\mathcal{E}}\right)=\left[p_{\tau}\left(\tau_{\mathcal{E}} \mid v_{1}, v_{2}\right)\right]$ for all possible values of $v_{1}$ and $v_{2}, p_{\tau}\left(\tau_{\mathcal{E}} \mid v_{1}, v_{2}\right)$ is given by equation (11). Then the posterior is given by vector $V_{1}^{+}$is computed as follows:

$$
V_{1}^{+} \propto V_{1} \circ\left(M\left(v_{\mathcal{E}}\right) V_{2}\right)
$$

where $\circ$ is the element-wise product (Hadamard product). $V_{1}^{+}$is obtained by renormalizing the right hand of the expression. The node $U_{2}$ performs the same evaluation steps. Once $U_{1}$ computed its posterior distribution $V_{1}^{+}$, it broadcasts it to its neighbors, hence to be considered int the next velocity estimation round when another "broken link" event occurs. To evaluate the effectiveness of the Bayesian inference with such limited information, we implement Bayesian inference (with python) and test it on a simple scenario with 2 nodes, one with speed $=1$ (expressed in unit distance per unit time, with unit distance defined by radio range $R=1$ ), and one with speed $=2$, with random (uniform) changes of direction 
between encounter. Initially, all the nodes are unaware of their real velocity values and use an arbitrary prior distribution (as common in Bayesian inference). Figure 1 represents the distribution of the velocity $v_{1}$ of one node: iteration number increases corresponding to encounters (and events of link breakage) with the other node. As illustrated, after 100 events, the distribution of the velocity is concentrated around its actual value 1 , indicating excellent convergence of the estimate.

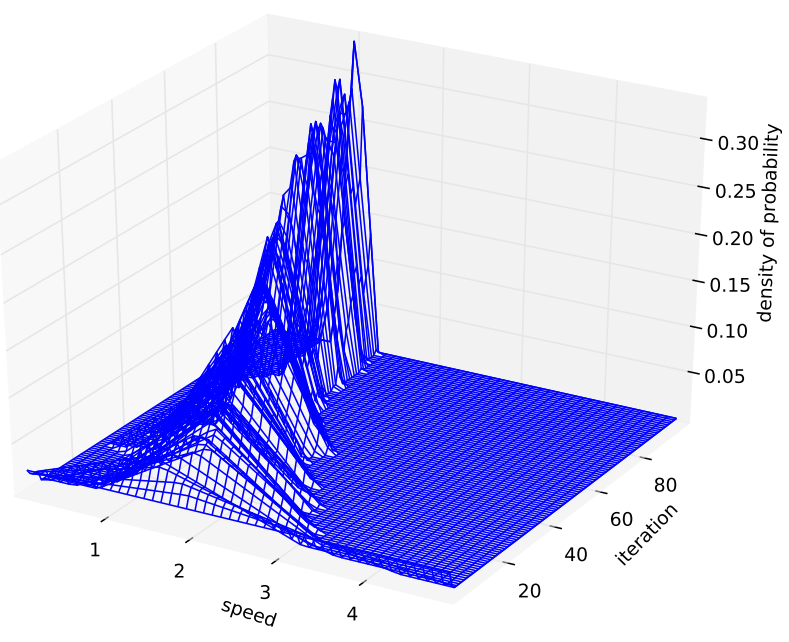

Fig. 1. Bayesian model validation

Despite its accuracy, the model proposed here requires exchanging a large amount of data, upon a "link expiration" event, corresponding to posterior link velocity estimation vectors. The overhead introduced by this exchanged information may largely degrade the network performance especially for high mobility scenarios. A simplification of the Bayesian inference model is detailed in the next section.

\section{SiMPLIFIED MOdEL}

A frequent exchange of posterior speed vectors computed by the Bayesian inference model may result in a non negligible overhead, hence increasing energy consumption in the network. Therefore, to reduce the amount of data sent upon the occurrence of expiration links events, we propose to model speed distributions as Gaussian distributions. Thus we send mean and standard deviation of (posterior) speed distributions. Receivers will interpret them as parameters of Gaussian distributions. Hence, once a node $U_{i}$ computes its posterior speed distribution vector $V_{i}^{+}$, then it evaluates the mean $\mu_{V_{i}^{+}}$and the standard deviation $\sigma_{V_{i}^{+}}$values and send them to its neighbors. The receiver reconstructs the pdf of the posterior distribution as:

$$
p_{v_{i}}^{+}\left(v_{i}\right)=\frac{1}{\sigma_{V_{i}^{+}} \sqrt{2 \pi}} e^{\frac{-1}{2}\left(\frac{v_{i}-\mu_{V_{i}^{+}}}{\sigma_{V_{i}^{+}}}\right)^{2}}
$$

From equation (14), we derive discretized values of speed distribution $p_{v_{i}}^{+}\left(v_{i}\right)$. The simplified model is validated (in
Python) using the same simulation procedure as in Figure 1. Mobile nodes may have one of the 5 velocities: $1,1.5,2,3$ and 4 . After 100 iterations, the final speed density curves are presented in Figure 2. The Figure shows that the estimated velocity values are very close to the real ones.

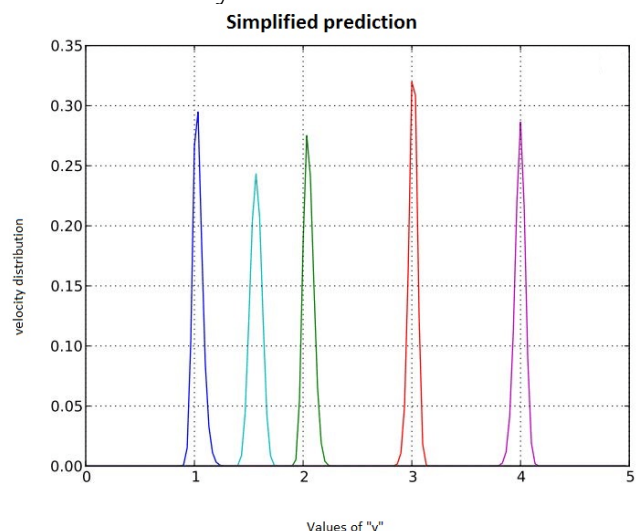

Fig. 2. The simplified model using 5 velocities

Moreover, to show the quick convergence of the speed estimation process, we depict in Figure 3 the average estimated speed values as a function of the iteration number. We also depict speed deviation curves given by (average speed +/standard variation). We notice, in Figure 3 , that the gap between curves decreases as the iteration number increases. This behavior highlights the fact that the predicted velocity converges to the real one.

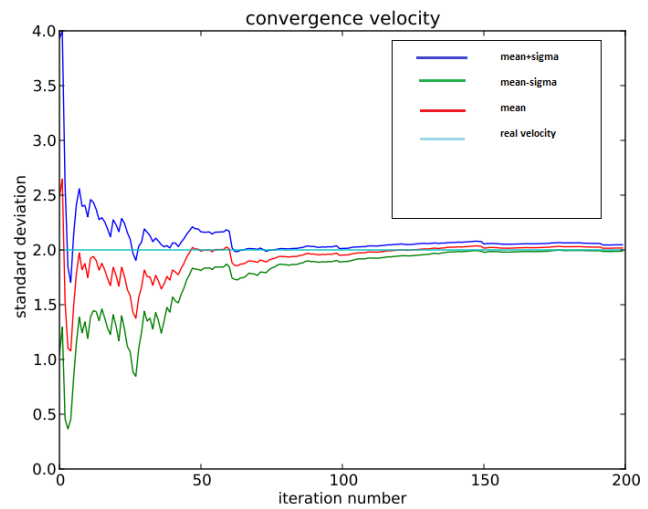

Fig. 3. Velocity behavior as a function of the iteration number

In Figure 4, we consider a large scale scenario with 50 mobile nodes and two velocity values ( 1 and 2 - half of the nodes each). We depict in Figure 4(a) (respectively in Figure 4(b)) the estimated average, maximum and minimum velocities of all mobile nodes with real speed 1 (respectively 2). Figures 4(a) and 4(b) show that all mobile nodes' predicted velocities (average, maximum and minimum) converge to real speed values 1 respectivly 2 .

The slower speed convergence is caused by the choice of the worst predicted values at each iteration (the minimum and the maximum values). Figure 4 illustrates the scalability of 


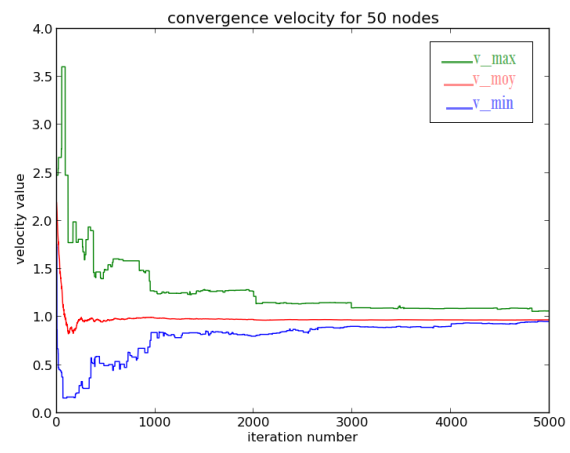

(a) Nodes with velocity 1

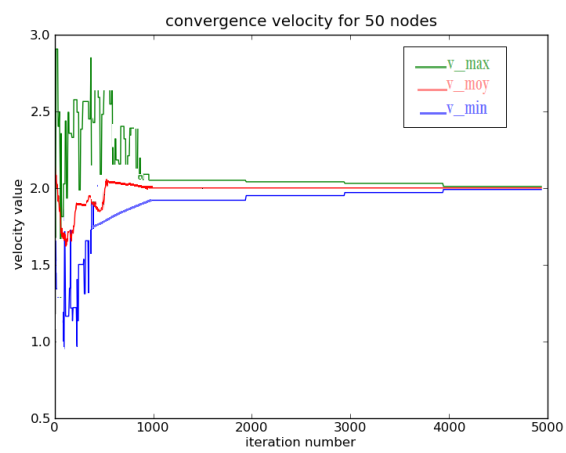

(b) Nodes with velocity 2

Fig. 4. Velocity convergence of the large scale mobility scenario

our mobility prediction model and shows that the Bayesian inference remains feasible for large-scale networks.

A further validation step is done in Figures 5 and 6 where realistic mobility models are used to derive link durations. Mobility scenarios are generated using the BonnMotion tool [12]. Analysis of the mobility scenario allows deriving links' duration values. These durations are therefore injected in the Bayesian model to predict nodes' velocities.

In Figure 5, we use the Random Waypoint (RWP) model to generate movement traces of two nodes with real velocity values equal to 0.9 and 1.8 . Figure 5 shows that with real mobility scenario, the Bayesian inference model converges after the given iteration (encounter) number 100. The same result is obtained in Figure 6 where the Random Direction (RD) mobility model is used and for sensor nodes speeds equal to 0.7 and 2 .

We can therefore conclude that all the performance results showed the efficiency of the Bayesian inference in predicting real sensor nodes' speed values and its convergence in a timely manner given unknown initial speeds; even with as minimal information as observed link durations.

\section{CONCLUSIONS AND Future WORK}

In this paper, we proposed a new mobility prediction model for WSNs based on a Bayesian inference approach. We discussed the existing solutions in MANETs and WSNs related to the nodes' mobility estimation. We then proposed a Bayesian

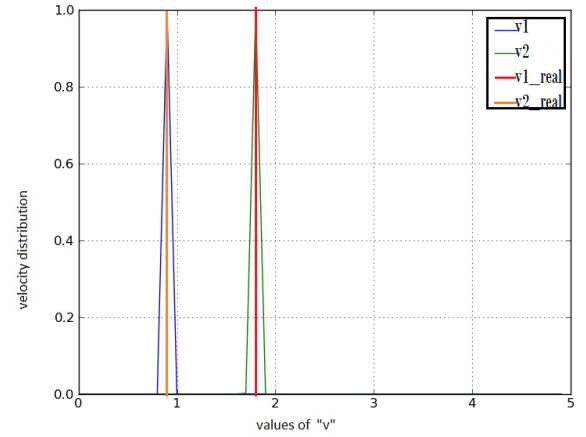

(a) final velocity values

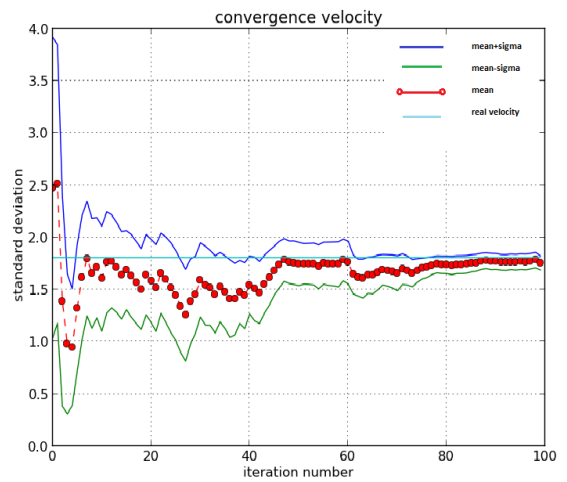

(b) intermediate velocity values

Fig. 5. Velocity convergence using the Random Waypoint (RWP) model

inference framework that derives sensor nodes' velocity only based on the occurrence of particular "link expiration" events. Therefore, we detailed the different steps of our mobility prediction model and simplified the model expressions hence to be easily implemented in a sensor network context. Performance evaluation results show the accuracy of the Bayesian Inference framework and its convergence in a timely manner.

In our future work, we intend to use the Bayesian inference model in the RPL protocol [12] to derive a new routing metric adapted to mobile sensor networks.

\section{REFERENCES}

[1] A. Somov, C. Dupont, and R. Giaffreda "Supporting smart-city mobility with cognitive internet of things", Future Network and Mobile Summit,2013.

[2] C. Peng, Y. Xiao and S. Youxian, "Distributed collaborative control for industrial automation with wireless sensor and actuator networks",IEEE Trans Ind Electron,2010.

[3] D. Culler, D. Estrin and M. Srivastava, "Overview of sensor networks",IEEE Comput,2004.

[4] D.Reina1, S. Toral1, P. Johnson and F. Barrero1 "Route duration improvement in wireless sensor and actuator networks based on mobility parameters and flooding control", EURASIP Journal on Wireless Communications and Networking, 2012.

[5] D. Ballari and chowicz, "The design of a Bayesian Network for mobility management in Wireless Sensor Networks",sixth international conference on Geographic Information Science,2010.

[6] I. F. Akyildiz and W. Wang, "The Predictive User Mobility Profile Framework for Wireless Multimedia Networks", IEEE/ACM Transactions on Networking,2004. 


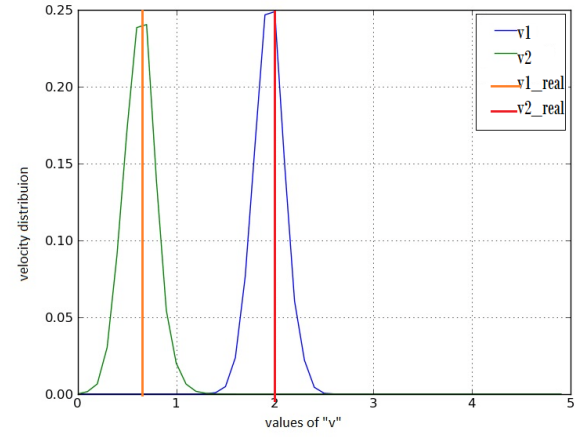

(a) final velocity values

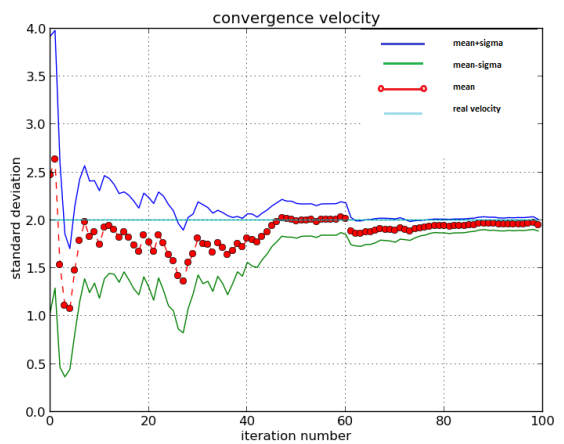

(b) intermediate velocity values

Fig. 6. Velocity convergence using the Random Direction(RD) model

[7] J. Ananthi and V. Ranganathan "A Review: On Mobility Prediction for Wireless Networks" International Journal of Emerging Technology and Advanced Engineering, 2013.

[8] K. C. Lee,R. Sudhaakar, L. Dai, S. Addepalli and M. Gerla, "RPL Under Mobility", IEEE Consumer Communications and Networking Conference (CCNC), 2012.

[9] L. Hou, K. Yeung and K. Wong, "Link Availability Prediction for Cognitive Radio Ad Hoc Networks", Journal of Computer and Communications, 2014.

[10] L. Meng, W. Fu, Z. Xu, J. Zhang and J. Hua, "A Novel Ad hoc Routing Protocol Based on Mobility Prediction", Information technology journal, 2008.

[11] N. Patwari, J.N. Ash, S. Kyperountas, A.O. Hero, RL Moses and NS Correal, "Locating the nodes. Cooperative localization in wireless sensor networks", Signal Process Mag,2005

[12] N. Aschenbruck, R. Ernst, E. Gerhards-Padilla, and M. Schwamborn, "BonnMotion - a Mobility Scenario Generation and Analysis Tool", in Proc. of the 3rd International ICST Conference on Simulation Tools and Techniques (SIMUTools), 2010.

[13] P. Samar and S. B. Wicker "On the behavior of communication links of a node in a multi-hop mobile environment", MobiHoc, 2004.

[14] P. Samar and S. Wicker, "Link Dynamics and Protocol Design in a Multi-Hop Mobile Environment", IEEE Transactions on Mobile Computing, 2006.

[15] R. A. Pushpa, A.Vallimayil and V.R.S. Dhulipala, "Impact of Mobility Models on Mobile Sensor Networks" International Journal of Communication Network and Security, 2011.

[16] S. Jiang, D. He, and J. Rao, "A prediction-based link availability estimation for routing metrics in manets", IEEE IACM Transactions on Networking, 2005.

[17] T. Fukushima, "Fast Computation of Complete Elliptic Integrals and Jacobian Elliptic Functions", National Astronomical Observatory of Japan 2-21-1, Japan

[18] V. Vasanthi, M. R. Kumar, N. A. Singh AND M.Hemalatha, "A Detailed Study of Mobility Models in Wireless Sensor Networks", Journal of Theoretical and Applied Information Technology, 2011.
[19] Y. Korilis, C. St-Jean, D. DeBarr, B. Carpenter and J. Chu-Carroll, "Modèles de Markov, Laboratoire d'Informatique Fondamentale de Lille, 2014.

[20] M. Grossglauser and D. Tse, "Mobility Increases the Capacity of Ad Hoc Wireless Networks", IEEE/ACM TRANSACTIONS ON NETWORKING, 2002.

[21] T. Winter, P. Thubert, A. Brandt, T. Clausen, J. Hui, R. Kelsey, P. Levis, K. Pister, R. Struik and J.Vasseur, $\hat{a} \in R P L$ : IPv6 Routing Protocol for Low power and Lossy Networksâ€, RFC 6550, IETF ROLL WG, March 2012

[22] JP. Vasseur, et al., ” Routing Metrics Used for Path Calculation in LowPower and Lossy Networks ", RFC 6551, IETF, 2012.

[23] C. Adjih, T. Plesse, "A Multi-Level Approach to Link State: MLOLSR", Proceedings of the 10th ACM international symposium on Mobility management and wireless, 2012 .

[24] Kevin C. Lee, Raghuram Sudhaakar, Jianxia Ning, Lillian Dai, Sateesh Addepalli,J. P. Vasseur, and Mario Gerla "A Comprehensive Evaluation of RPL under Mobility", Hindawi Publishing Corporation International Journal of Vehicular Technology , 2012.

[25] I. El Korbi, M. Ben Brahim, C. Adjih and L. Saidane, "Mobility Enhanced RPL for Wireless Sensor Networks", Network of the future,2012.

[26] G. Box and G. Tiao, "Bayesian inference in statistical analysis", John Wiley and Sons, 25 janvier 2011, 608 pages.

[27] W. Su, S.-J. Lee, and M. Gerla, "Mobility Prediction and Routing in Ad Hoc Wireless Networks", Int'l J. Network Management, vol. 11, no. 1 , pp. 3-30, Jan./Feb. 2001.

[28] M. Gerharz, C. de Waal,, M. Frank, and P. Martini, "Link Stability in Mobile Wireless Ad Hoc Networks", Proc. IEEE Conf. Local Computer Networks, Nov. 2002.

[29] T. M. Mitchell, "Bayesian Learning," in Machine Learning, McGrawHill, 1997, pp. 154 - 200.

[30] T. The Son, H. Le Minh, G. Sexton, N. Aslam and Z. Ghassemlooy, "Bayesian Model for Mobility Prediction to Support Routing in Mobile Ad-Hoc Networks" in Proc of the 24th IEEEE International Symposium on Personal, Indoor and Mobile Radio Communications, PIMRC'13, UK, September 2013. 